\title{
Nitric oxide synthase-like protein in pea (Pisum sativum L.)
}

\begin{abstract}
Nitric oxide synthase activity was detected in pea (Pisum sativum L.) leaf extracts using a citrulline formation assay that is typically employed in mammalian systems. A total protein extraction method was modified from that used in mammalian systems based on biochemical activities such as the use of protease inhibitors, $\mathrm{pH}$, and precipitation with salts and organic solvents. Physiological aspects in plants, such as effects of chemicals that induce systemic resistance to NOS activity and immunodetection of an NOS-like protein, were also studied. The NOS-like protein was partially isolated using liquid chromatography and characterized based on mammalian NOS inhibitor and cofactor requirements. Correlation of NOS activity and NOS-like gene expression during incompatible and compatible peaï bacteria interactions were investigated using interactions of Ralstonia solanacearum and Pseudomonas syringae pv. pisi, respectively, with pea. NOS activity was detected using citrulline formation assay. Gene expression was measured using real-time reverse transcription-polymerase chain reactions and a 348-bp probe designed from a cloned cDNA fragment of pea that was homologous to NOS of snail and AtNOS1/AtNOA1 of Arabidopsis. The possibility of NO production from various sources in cells of pea is also discussed.
\end{abstract}

Keyword: Pea; Nitric oxide synthase (NOS); Ralstonia solanacearum; Pseudomonas syringae pv pisi 